\title{
Noise and physical limits to maximum resolution of PET images
}

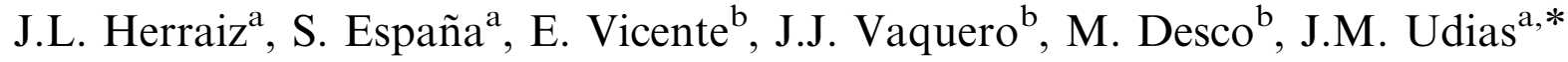 \\ ${ }^{a}$ Dpto. Física Atómica, Molecular y Nuclear, Facultad de Ciencias Físicas, Universidad Complutense de Madrid, Avda. Complutense s/n, \\ E 28040 Madrid, Spain \\ ${ }^{\mathrm{b}}$ Unidad de Medicina y Cirugía Experimental, Hospital GU “Gregorio Marañón”, E 28007 Madrid, Spain
}

\begin{abstract}
In this work we show that there is a limit for the maximum resolution achievable with a high resolution PET scanner, as well as for the best signal to noise ratio, which are ultimately related to the physical effects involved in the emission and detection of the radiation and thus they cannot be overcome with any particular reconstruction method. These effects prevent the spatial high frequency components of the imaged structures to be recorded by the scanner. Therefore, the information encoded in these high frequencies cannot be recovered by any reconstruction technique. Within this framework, we have determined the maximum resolution achievable for a given acquisition as a function of data statistics and scanner parameters, like the size of the crystals or the inter crystal scatter. In particular, the noise level in the data as a limitation factor to yield high resolution images in tomographs with small crystal sizes is outlined. These results have implications regarding how to decide the optimal number of voxels of the reconstructed image or how to design better PET scanners.
\end{abstract}

Keywords: Positron emission tomography (PET); Image quality; Monte Carlo application

\section{Introduction}

Dedicated small PET animal scanners have become one of the main tools in molecular imaging research. In this kind of studies, spatial resolution of $1 \mathrm{~mm}$ and high sensitivity are desired. Iterative statistical reconstruction methods [1] have shown superior image quality to that provided by conventional analytic reconstruction techniques, but despite their widespread and now conventional use, the limit of resolution achievable by these methods on high-resolution scanners has not been quantitatively well assessed.

The Point Spread Function (PSF) has been traditionally employed for estimating the resolution of a tomograph [2]. Nevertheless, measurement of the PSF is not free from problems. On the one hand, it involves the reconstruction of a source, and therefore the impact of the reconstruction method chosen on the PSF has to be properly taken into account [3]. On the other hand, the PSF is usually evaluated from high activity point sources or simulations with a very high statistics. Consequently, the influence of noise in the resolution is hardly considered on the PSF and therefore the results obtained may not be realistic for real studies.

The majority of available PET scanners are configured as small individual detector units constructed around some type of scintillator crystal array. Bearing in mind all the physical effects (positron range, non-collinearity, scatter of the gamma rays inside the object and inside the crystals) and electronic noise involved in the data acquisition of a PET scanner, the volume of the space from which an emitted positron can produce coincidence events in a pair of crystals is distributed on a wide 'tube of response' (TOR) along the line of response (LOR). In high-resolution PET scanners, with small crystal sizes, TORs are considerably wider than what is obtained from pure geometrical considerations. In a previous work we have shown [4] that the size and shape of the TORs show a 'blurring' effect on the acquired data that determines the resolution of the 
scanner. This effect is more easily described in the frequency domain. If we define the modulation transfer function (MTF) as the Fourier transform of the probability distribution of the TORs, we may show that the frequencies of the data and the object are related as

Freq $($ Data $)=$ Freq $($ Object $) \times$ MTF + Noise

In this work, we review the issue of the maximum resolution achievable in a particular PET acquisition with a given scanner. We establish that it is more related to the physical effects involved in the emission and detection of the radiation and the acquisition statistics than to the particular iterative reconstruction method chosen.

We have determined the maximum resolution achievable as a function of the FWHM of the transversal gaussian shape of the TORs and the noise level, independently of the reconstruction method employed. Finally, based on these results, we also propose criteria for the design of scanners, optimized for statistical reconstruction methods bearing in mind realistic acquisition times and activities.

\section{Methods}

The maximum resolution achievable for a PET scanner is determined by the maximum system frequency (MSF), defined as the highest frequency of the object that the scanner can record in the ideal case of a noise-free acquisition. An analytical estimation of the MSF can be obtained as follows:

We assume that every TOR has a transversal gaussian shape (Fig. 1), all of them with the same full-width at halfmaximum (FWHM). For the sake of simplicity, only the one-dimension case is discussed here. Therefore, the probability distribution of a TOR can be expressed as $C(r)=\exp \left(-r^{2} / 2 \sigma^{2}\right), r$ being the radial variable of the sinogram. The MTF will be given by its normalized Fourier transform: $G(w)=\exp \left(-\sigma^{2} w^{2} / 2\right)$, and using the relation $w=2 \pi f$, we may rewrite it as: $G(f)=$ $\exp \left(-2(\pi \sigma f)^{2}\right)$. Now, if we define the MSF to be at the half-width at tenth-maximum (HWTM) of this MTF, it may be deduced that:

$$
\begin{aligned}
\operatorname{MSF}\left(\mathrm{mm}^{1}\right) & =\frac{\sqrt{ } 2 \operatorname{Ln} 10}{2 \pi \sigma(\mathrm{mm})} \approx \frac{1}{3 \sigma(\mathrm{mm})} \\
\rightarrow \Delta x(\mathrm{~mm}) & =\frac{1}{2 \operatorname{MSF}\left(\mathrm{mm}^{1}\right)} \approx \frac{3}{2} \sigma(\mathrm{mm}) .
\end{aligned}
$$

This result means that in an ideal noise-free acquisition, the best resolution achievable with a PET scanner can be estimated to be $3 / 2$ times the standard deviation of the gaussian that we have used to fit the probability distribution of the TORs.

This also implies that, in order to avoid noise aliasing, the optimum voxel size of reconstructed images is $1 / 2$ of this value.

When noise is present, an effective maximum system frequency (EMSF) can also be defined, as the highest frequency of the object that can be recorded above the noise level. The EMSF can be estimated under the assumption that the power spectrum of the noise is flat

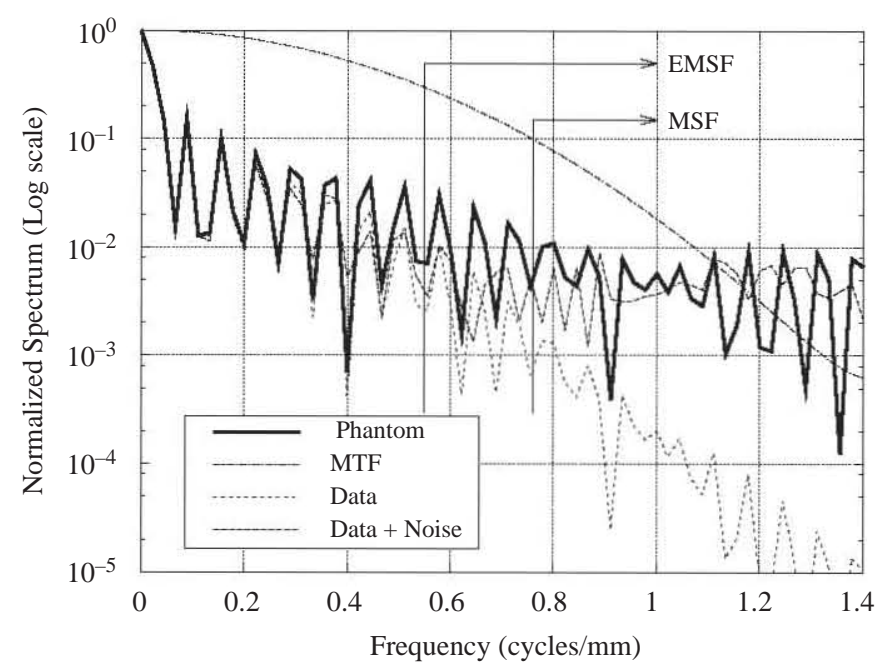

Fig. 2. Frequency spectrum corresponding to a simulated one dimen sional case. The frequencies of the noise free acquisition correspond to the product of the frequencies of the phantom and the MTF of the tomograph. It can be noticed that in the noisy data those frequencies higher than the EMSF are dominated by noise.
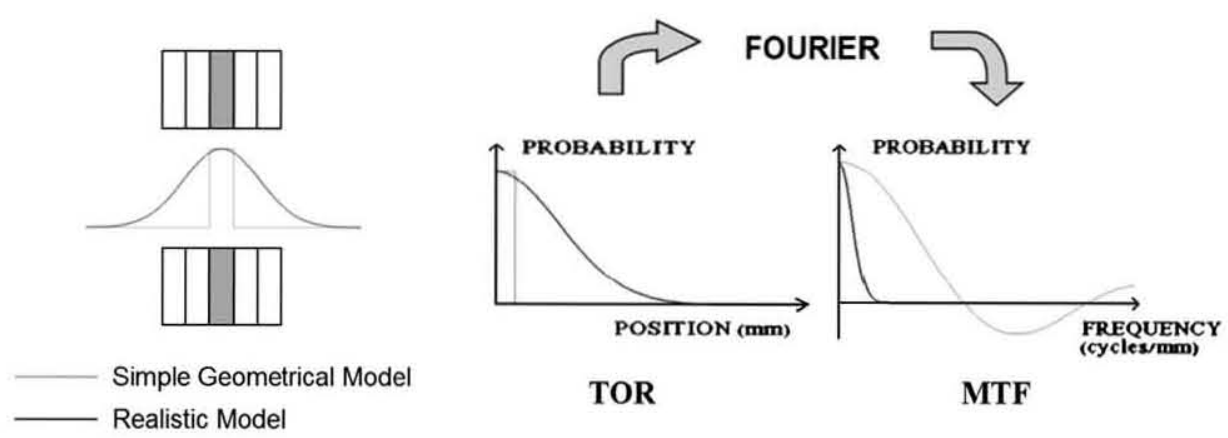

Fig. 1. Schematic tubes of response (TOR) and their corresponding modulation transfer function (MTF) in frequency domain. 
and thus dominates in the high frequency region (Fig. 2). It should be noted that, in high-resolution tomographs, the EMSF is more restrictive than the Nyquist Frequency (in Fig. 2 it corresponds to the higher frequency represented). Therefore, aliasing is not the main limiting factor.

In this work, we have obtained the EMSF for a simulation of realistic acquisitions of a point source inside a water cylinder, located in different places along a line equidistant to a pair of block detectors. Therefore, all the considered LORs are perpendicular to the scintillator crystals. The activity of the source and the acquisition time was chosen to produce similar number of events per LOR as in a FDG mouse study [5]. The simulations were performed with PeneloPET, a Monte Carlo simulation code developed by our group based on PENELOPE [6]. A small animal PET scanner $(\mathrm{FOV}=50 \mathrm{~mm}$ diameter $)$ with $2 \mathrm{~cm}$-long LYSO crystals were simulated considering the main physical effects: positron range $\left({ }^{18} \mathrm{~F}\right.$ in water), non-collinearity, interaction of photons at scintillator crystals, anger logic and electronics.

\section{Results and discussion}

Fig. 3 shows the MTF of the simulated PET scanner corresponding to different crystal sizes, i.e. increasing the granularity of the block detectors. These results are in agreement with those obtained by Stickel and Cherry [7].

Nevertheless, reducing the pixel size decreases the sensitivity of each particular LOR, raising the noise level. For a typical activity and acquisition time the impact of statistical Poisson noise on the highest resolution achievable has been evaluated. Numerical estimations of the EMSF from these simulations are summarized in Table 1.

These results show that reducing the crystal size improves the MSF but at the cost of an increase of the noise level. As is shown in Table 1, for the activities commonly used in real studies, beyond certain pixel size, noise becomes the main limiting factor to the highest achievable resolution.

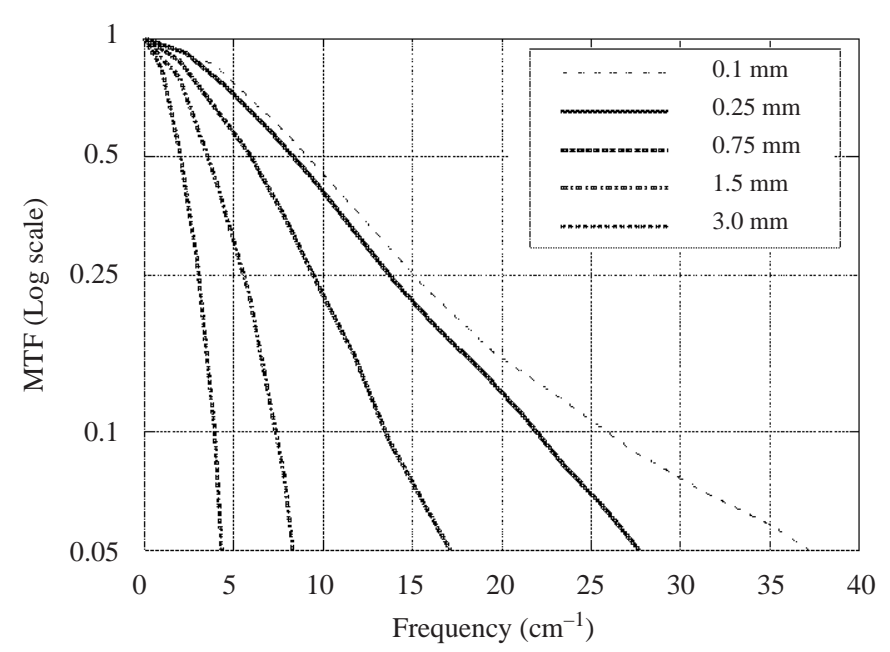

Fig. 3. MTF as a function of crystal size.
Table 1

MSF, number of counts per LOR, EMSF and best resolution achievable for a fixed activity and acquisition time as a function of the pixel size

\begin{tabular}{lllll}
\hline $\begin{array}{l}\text { Pixel size } \\
(\mathrm{mm})\end{array}$ & $\begin{array}{l}\text { MSF } \\
\left(\mathrm{mm}^{1}\right)\end{array}$ & $\begin{array}{l}\text { Counts per } \\
\text { LOR }\end{array}$ & $\begin{array}{l}\text { EMSF } \\
\left(\mathrm{mm}^{1}\right)\end{array}$ & $\begin{array}{l}\text { Resolution } \\
(\mathrm{mm})\end{array}$ \\
\hline 3.00 & 0.40 & 944 & $>\mathrm{MSF}$ & 1.25 \\
1.50 & 0.75 & 100 & $=\mathrm{MSF}$ & 0.66 \\
0.75 & 1.35 & 11 & 0.80 & 0.62 \\
0.50 & 1.75 & 3 & 0.75 & 0.66 \\
0.25 & 2.20 & 0.30 & & \\
\hline
\end{tabular}

This work shows the importance of developing a theoretical framework for evaluating the impact of the physical effects involved in a PET data acquisition (including statistical noise) on the maximum resolution achievable. This would allow us to establish criteria on some open issues in PET reconstruction, such as the optimum voxel size of the reconstructed images or the optimum size of the scintillator crystals for a positron emission tomograph.

\section{Conclusions}

Analysis of PET-projection data in the frequency domain combined with an accurate model of the system can be used to estimate the best achievable resolution for a particular acquisition.

It has been shown that, in real studies, statistical noise plays an important role in the resolution that should not be neglected. Furthermore, with our proposed theoretical framework, the impact of noise on the resolution can be estimated.

These results have implications for the design of small animal PET scanners. Reducing the crystal size improves the MTF but at the cost of increasing the noise level. Therefore, for a given source activity, the EMSF cannot be improved beyond a certain point.

\section{Acknowledgements}

We acknowledge support from the CD-TEAM project of the CENIT program, and CDTI J.L. Herraiz acknowledges support from UCM grant. J.M. Udias acknowledges support from MEC (BFM2003-04147-C02-01), E. Vicente acknowledges support from "Fund. para la investigación biomédica del Hospital Gregorio Marañon" grant, J.M. Udias, J.L Herraiz and S. España acknowledge support from MEC under FPA2006-07393 and FIS (PI052583) projects.

\section{References}

[1] R.M. Lewitt, S. Matej, Proc. IEEE 91 (2003) 1588.

[2] D. Schmitt, B. Karuta, C. Carrier, R. Lecomte, IEEE Trans. 7 (1988) 2. 
[3] V. Frouin, C. Comtat, A. Reilhac, A.C. Evans, M.C. Gregoire, in: Proceedings of the IEEE Nuclear Science Symposium and Medical Imaging Conference, vol. 3, 2000, p. 18/57.

[4] J.L. Herraiz, S. Espana, J.M. Udias, J.J. Vaquero, M. Desco, in: Proceedings of the IEEE Nuclear Science Symposium and Medical Imaging Conference, vol. 4, 2005, p. 1846.
[5] J.L. Herraiz, S. Espana, J.J. Vaquero, M. Desco, J.M. Udias, Phys. Med. Biol. 51 (2006) 4547.

[6] J. Sempau, J.M. Fernandez Varea, E. Acosta, F. Salvat, Nucl. Instr. and Meth. B: Beam Interact. Mater. Atoms 207 (2003) 107.

[7] J.R. Stickel, S.R. Cherry, Phys. Med. Biol. 50 (2005) 179. 